\title{
Exploiting entropy masking in perceptual graphic rendering
}

\author{
Lu Dong ${ }^{\mathrm{a}}$, Yuming Fang ${ }^{\mathrm{b}}$, Weisi Lin ${ }^{\mathrm{a}}$, Chenwei Deng ${ }^{\mathrm{c}}$, Ce Zhu ${ }^{\mathrm{d}}$, Hock Soon \\ Seah $^{\mathrm{a}}$ \\ ${ }^{a}$ School of Computer Engineering, Nanyang Technological University, Singapore \\ ${ }^{b}$ School of Information Technology, Jiangxi University of Finance and Economics, China \\ ${ }^{c}$ School of Information and Electronics, Beijing Institute of Technology, China \\ ${ }^{d}$ School of Electronic Engineering, University of Electronic Science and Technology of \\ China, China
}

\begin{abstract}
Since the human visual system (HVS) is the ultimate appreciator of most photorealistically rendered images, rendering process can be accelerated by exploiting the properties of the HVS. According to the concept of entropy masking, the HVS is not sensitive to visual distortions in unstructured visual signals. For structured regions, pixels are highly correlated, while the similarity among pixels in unstructured regions is low. In this paper, we detect unstructured regions by extracting local patches from each pixel and its neighboring pixels, and comparing the similarity between the local patches of the center pixel and the neighboring pixels. We further exploit entropy masking in perceptual rendering, and experimental results demonstrate that the proposed method can accelerate rendering, without degrading the perceived quality of resultant images.
\end{abstract}

Keywords: Entropy masking, unstructured visual signal, graphic rendering

\section{Introduction}

Physically-based rendering produces photorealistic images by simulating the physical behavior of light interaction with surfaces and volumes, and

Email addresses: s090059@e.ntu.edu.sg (Lu Dong), fa0001ng@e.ntu.edu.sg (Yuming Fang), wslin@ntu.edu.sg (Weisi Lin), cwdeng@bit.edu.cn (Chenwei Deng), eczhu@uestc.edu.cn (Ce Zhu), ashsseah@ntu.edu.sg (Hock Soon Seah) 
this process is usually time-consuming [1]. Since the human visual system (HVS) is the ultimate appreciator of most photorealistic images, significant computation can be saved by exploiting properties of the HVS [2]. Among various properties, visual masking refers to the decrease in visibility to a visual signal in the presence of image background [3]. That means we can use fewer computational resources in rendering, since low-level artifacts are not visible in regions with strong masking effect.

Existing studies on visual masking include contrast masking and entropy masking [4]. Contrast masking refers to the variation of visibility of visual signals due to the background contrast [5]. Entropy masking refers to the decrease of visibility to a visual signal imposed on a mask signal which is unfamiliar or uncertain to human eyes [6]. For each pixel in an image, the entropy masking effect depends on the structure complexity of a neighborhood [4]. Unstructured regions are less predictable and more difficult to be learned than structured regions, and thus have strong entropy masking effect [7].

Existing studies on entropy masking $[4,8,9]$ try to estimate the masking effect using Shannon entropy [10]. Given a pixel $x_{o}$, a luminance histogram is first constructed from pixels in the $m \times m$ local patch $P_{x_{o}}$ centered at the pixel $x_{o}$. The entropy $H\left(P_{x_{o}}\right)$ of patch $P_{x_{o}}$ centered at the pixel $x_{o}$ is calculated as:

$$
H\left(P_{x_{o}}\right)=-\sum_{i=0}^{M} p\left(l_{i}\right) \cdot \log p\left(l_{i}\right)
$$

where $l_{0}, \ldots, l_{M}$ are luminance values of pixels in patch $P_{x_{o}}$, and $p\left(l_{i}\right)$ is the occurrence rate of luminance value $l_{i}$ in patch $P_{x_{o}}$. From Eq. 1, the entropy of an image patch mainly depends on the relative occurrence of pixel values in the patch regardless of the patch structure. With the Shannon entropy, the computed masking effect of an unstructured patch and a structured patch is the same, if these two patches share the same histogram.

The study [11] proposes a method to predict pattern complexity. In the proposed method, unstructured and structured patterns corresponds to irregular and regular patterns mentioned in the study [11], respectively. The method [11] is designed based on a gradient count. If the difference between the colors of the pixel and its adjacent pixels exceeds a predefined threshold, the gradient count is one, otherwise, the gradient count is zero. A higher value of pattern complexity is assigned to texture with larger sum of gradient counts. According to the method [11], the gradient counts are zero for pixels 
in smooth patterns, and the gradient counts are one for pixels in dense, mixed color patterns, and edges. Therefore, the method [11] is able to distinguish smooth patterns from dense and mixed color patterns; however, it cannot distinguish structured patterns from unstructured ones, since both structured and unstructured patterns include edges and the sum of gradient counts from edge pixels are high.

To distinguish structured regions from unstructured regions, we design a method by taking into account the spatial correlation among neighboring pixels. In structured regions, pixels are highly correlated with their neighboring pixels and similar local patterns can be detected in a neighborhood. For each pixel, we extract the local patches centered at the pixel as well as its neighboring pixels, and compute the similarity between the patches of the center pixel and the neighboring pixels. Then the value of the center pixel is predicted from the neighboring pixels with similar local patches to the center pixel. The predicted pixel value is treated as the structured signal, and the difference between the original pixel and the predicted one is treated as the unstructured signal, which indicates the masking effect of the original pixel.

Contrast masking has been exploited in perceptual graphic rendering [1215] to improve the efficiency. However, entropy masking has not been exploited in perceptual graphic rendering. In this work, we explore the entropy masking properties by proposing a perceptually adaptive sampling scheme. The proposed method distributes samples according to the level of noise as well as the entropy masking effect, and it substantially decreases the rendering cost.

The contribution of the proposed method is twofold. First, we propose a new formulation of entropy masking by detecting unstructured regions. Second, we exploit entropy masking in perceptual graphic rendering by adaptively distributing samples.

\section{Related work on perceptual graphic rendering}

Perceptual graphic rendering tries to improve rendering quality or decrease rendering computation by modeling properties of the visual perception. Bolin and Meyer [12] built a perceptual model for adaptive sampling by predicting the visible error between two intermediate rendering results, and iteratively distributing new samples to locations with the largest visible error. Ferwerda et al. [16] made an early attempt to take advantage of visual masking in computer graphics. Given a reference image and a distorted 
image, the method applies spatial frequency tuning and orientation tuning to each image, and computes the responses to different spatial frequency and orientation bands. The computed responses are modulated by the masking functions to account for the masking interactions among the spatial frequency components. The modulated responses of the reference image and the distorted image are compared to determine whether the two images are visually different. The method estimates the visual difference between a reference image and a distorted image but cannot estimate the masking effect of each pixel in the reference image. In the proposed method, we need to estimate the masking effect of each pixel and use the results to determine the number of pixels distributed to each pixel in adaptive sampling scheme.

Ramasubramanian et al. [17] proposed a computationally efficient vision model based on characteristics of the HVS, including luminance adaptation, spatiotemporal contrast sensitivity and visual masking. Given two intermediate rendered images, the vision model computes a threshold map with different visibility on the first image. The difference between the two images are also computed and compared against the visibility threshold map. In the next iteration, more computation is allocated to regions where difference is larger than the threshold. Myszkowski et al. [18] extended the use of human vision model to animation rendering. They developed the Animation Quality Metric (AQM) by extending an existing vision model called Visible Difference Predictor (VDP) [3]. Later they applied AQM to walk-through animation rendering [19] and global illumination computation for animation rendering [20]. A more detailed introduction of perceptual image rendering can be found in $[2,21]$.

All perceptual rendering algorithms mentioned above include the evaluation of a full human vision model during image synthesis. Computational complexity is a major concern in these perceptual rendering methods, since the vision models used are generally sophisticated and it is time-consuming to continuously update the vision models. Therefore, perceptual rendering methods concentrating on visual masking have been proposed by detecting regions with strong masking effect to decrease rendering computation in these regions. They aim to benefit from the perceptual properties of image content with less computational complexity.

The method [14] computes visual masking properties of image content by using components of JPEG compression standard. The assumption in [14] is that at moderate compression rates, the quantization errors in JPEG compression are largely unnoticeable, i.e. the error between the original and 
JPEG decoded images is less than a masking threshold of the image content. Visual masking effect is estimated as the difference between original and JPEG decoded images.

$\mathrm{Qu}$ and Meyer [15] proposed to compute the visual masking properties of image content based on a computational model of human vision. The method does not use the vision model to predict the difference between two intermediate rendering results and update the vision model during rendering as other perceptual rendering methods $[12,17]$ do. Instead, the method uses the vision model to estimate the masking effect of image content. Two maps are generated: one indicates the visual difference between the original image and a filtered one; while the other denotes the visual masking. These two maps are combined into a final map, which indicates the visual masking effect in the original image.

Contrast masking has been exploited in existing perceptual rendering methods, but entropy masking mechanism has remained unexplored in the area of graphic rendering. Considering that unstructured visual signals have strong masking to rendering artifacts, we explore how to make use of entropy masking in rendering acceleration.

Some studies have also investigated visual masking effect for 3D geometrical meshes. Lavoué [22], and Wang et al. [23] modeled visual masking based on local roughness of 3D mesh surfaces, and applied the model in mesh compression. Váša and Rus [24] proposed a mesh error metric based on visual masking and dihedral angle of triangle meshes. 3D based visual masking models estimate the masking effect of a vertex based on attributes of $3 \mathrm{D}$ meshes. We will investigate the masking effects in $3 \mathrm{D}$ in the future work.

\section{The proposed method}

\subsection{The proposed formulation of entropy masking}

In the proposed method, the entropy masking effect of an image is estimated by detecting the unstructured regions. Unstructured patterns exhibit strong visual masking of rendering artifacts. In these three low-quality images (Fig. 1(d) - (f)), the teapots are rendered with the same amount of computation, and thus have similar objective levels of rendering noise. As shown in this figure, the texture in Fig. 1(a) is smooth and the texture in Fig. 1(b) is structured, while the texture in Fig. 1(c) is unstructured. Compared with the high-quality reference images in Fig. 1(a) - (c), the noise in 


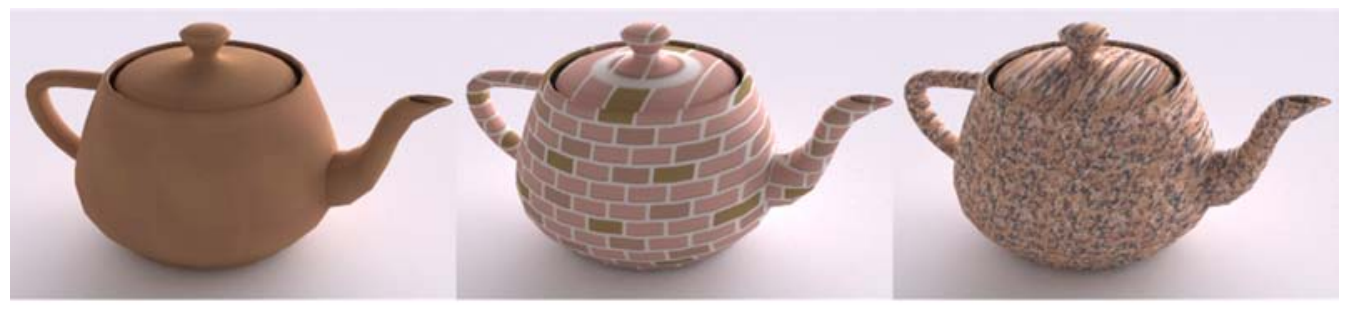

(a)

(b)

(c)

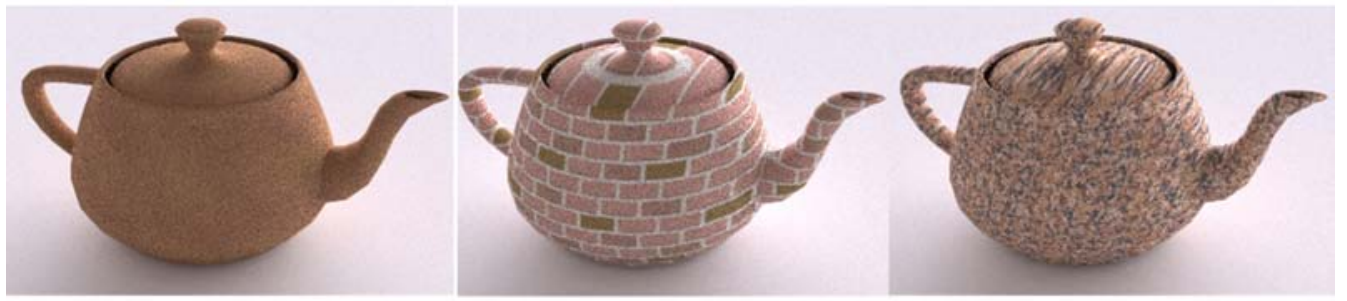

(d)

(e)

(f)

Figure 1: Examples of masking properties of different patterns. The top row: high-quality reference images; bottom row: low-quality rendered images.

Fig. 1(d) and (e) is noticeable. However, it is difficult to detect the noise in Fig. 1(f). This example illustrates the decrease in visibility of rendering noise in the presence of unstructured patterns.

Existing entropy masking formulations [4, 8, 9] compute entropy masking effect by using the Shannon entropy. However, since the spatial correlation among neighboring pixels is not considered when computing Shannon entropy, regions with the same entropy may have different levels of masking effect due to the difference in structure complexity. Fig. 2 shows two artificial patterns in (a) and (b). These two patterns have the same entropy value ' 1 ', with a half of the pixels being white and the other half being black. However, the noise in the contaminated pattern in (c) is visible while the noise in the contaminated pattern in (d) is masked by unstructured visual signals. Although pattern (b) is with the same Shannon entropy to pattern (a), pattern (b) is structurally more complex than pattern (a) and has stronger masking effect to noise.

To distinguish structured and unstructured regions, we explore the properties of these two types of regions. In structured regions, pixels are highly correlated with their neighboring pixels and similar local patterns can be detected. For instance, in Fig. 3(a) - (c), given a target patch highlighted with a red rectangle, neighboring patches with similar structure to the target 


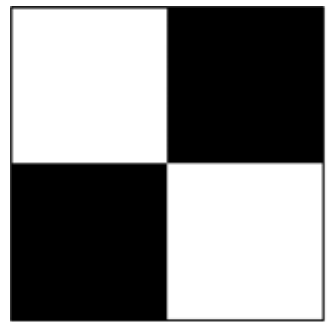

(a)

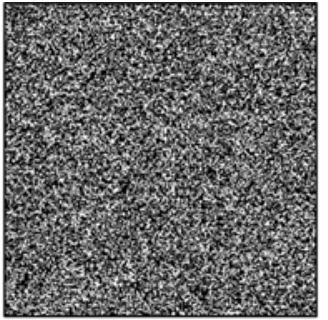

(b)

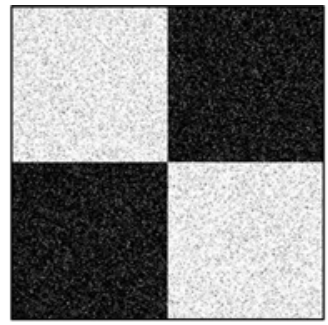

(c)

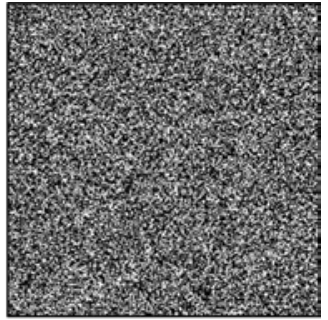

(d)

Figure 2: Patterns with the same entropy but different masking effect. (a) is a structured pattern; (b) is a non-structured pattern; (c) and (d) are two patterns generated by imposing the same noise pattern in (a) and (b), respectively.

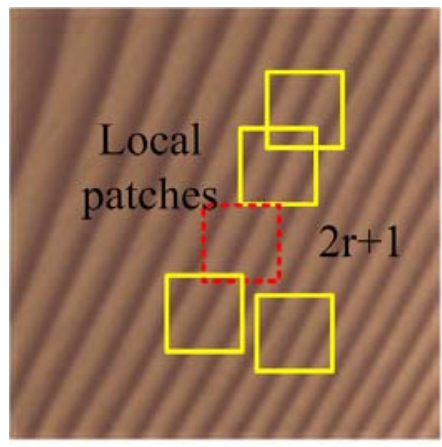

(a)

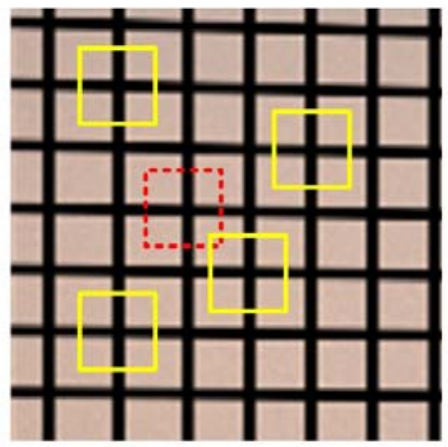

(b)

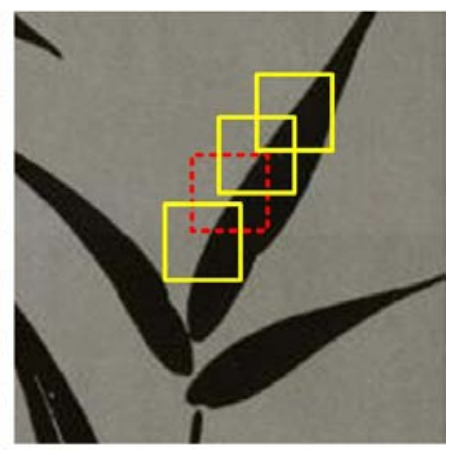

(c)

Figure 3: Structured regions include repetitive local patterns. (a), (b) and (c) are three structured regions, and a local $(2 r+1) \times(2 r+1)$ patch is highlighted with a red rectangle in the center of each image. Patches with similar structure to the center patch are highlighted with yellow rectangles.

patch (i.e. the patches highlighted with yellow rectangles) can be detected. On the other hand, it is difficult to find patches with similar structure in unstructured regions. Based on these properties, we propose a method to detect the unstructured regions. We extract local patches from each pixel, and compute the similarity between the local patches of the center pixel and the neighboring pixels. Then each pixel is predicted by neighboring pixels with similar local patches. The difference between the original and the predicted signals is used in estimating the visual masking effect of the pixel.

More specifically, starting from an input image, we use the luminance component (LAB color space) of the input image, since the HVS is more sensitive to the luminance component than to color components. For a pixel 


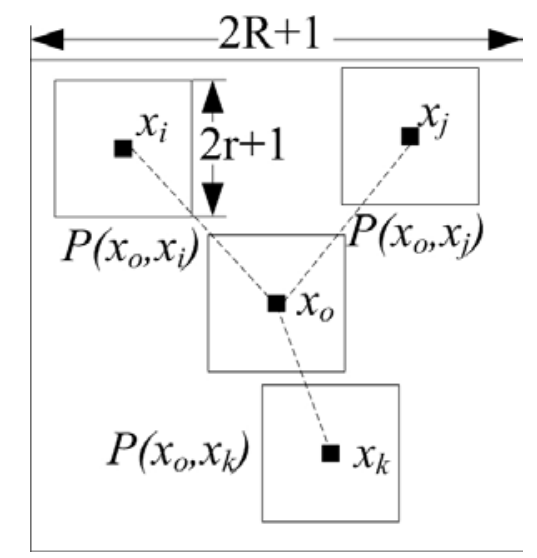

Figure 4: Illustration of the proposed scheme.

$x_{o}$ in the input image, a $(2 R+1) \times(2 R+1)$ neighborhood centered at this pixel is extracted, as illustrated in Fig. 4. The local patches $P$ s with the size $(2 r+1) \times(2 r+1)$ are also extracted from the neighboring pixels (e.g. $\left.x_{i}, x_{j}, x_{k}\right)$ as well as the center pixel $x_{o}$. The local patch distance $d_{P}\left(x_{o}, x_{i}\right)$ between a pair of center-surround patches $P_{x_{o}}$ and $P_{x_{i}}$ is calculated as the average of the per-pixel squared distances over the patches:

$$
d_{P}\left(x_{o}, x_{i}\right)=\frac{1}{(2 r+1)^{2}} \sum_{x_{n} \in P_{x_{i}}} \sum_{x_{m} \in P_{x_{o}}}\left(L_{x_{m}}-L_{x_{n}}\right)^{2}
$$

where $L_{x_{m}}$ and $L_{x_{n}}$ are the values of the pixels in patches $P_{x_{o}}$ and $P_{x_{i}}$, respectively. The similarity $S\left(x_{o}, x_{i}\right)$ between the patches is computed as:

$$
S\left(x_{o}, x_{i}\right)=\exp \left(-\frac{d\left(x_{o}, x_{i}\right)}{2 \sigma^{2}}\right)
$$

where $\sigma$ is a damping factor, and a higher value yields a stronger blurring effect. When $d\left(x_{o}, x_{i}\right)$ approaches positive infinity, the similarity $S\left(x_{o}, x_{i}\right)$ will approach " 0 "; while $d\left(x_{o}, x_{i}\right)$ equals to zero, the similarity $S\left(x_{o}, x_{i}\right)$ will be "1". A low $\sigma$ is expected in rough regions to preserve the structural information, while a higher $\sigma$ is expected in smooth regions to preserve the smooth appearance of the these regions. Therefore, we adopt the standard deviation $\operatorname{std}(\cdot)$ of the local patch, indicating whether a region is smooth or 


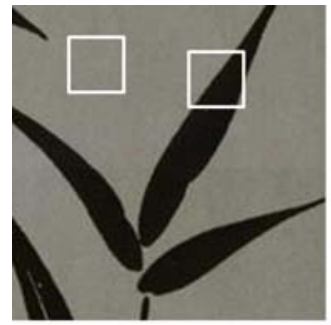

(a)

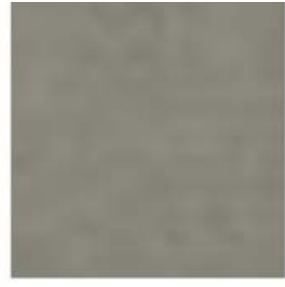

std $=2.3$

(b)

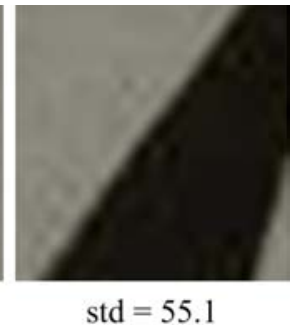

(c)

Figure 5: Standard deviation values of two patches. (b) and (c) correspond to two patches highlighted in (a).

not, to determine the $\sigma$ :

$$
\sigma= \begin{cases}\sigma_{t}, & s t d\left(P\left(x_{o}\right)\right) \leq \sigma_{t} \\ \sigma_{t} \cdot\left(\frac{\sigma_{t}}{\operatorname{std}\left(P\left(x_{o}\right)\right)}\right), & \text { std }\left(P\left(x_{o}\right)\right)>\sigma_{t}\end{cases}
$$

where $\sigma_{t}$ is set to 5 in the proposed method, since we have observed that patches with standard deviation equal or smaller than 5 are usually smooth ones. Fig. 5 (b) and (c) show two example patches and their standard deviation values.

After the difference computation, each pixel is predicted as a similarityweighted sum of the neighboring pixels:

$$
L_{x_{o^{\prime}}}=\frac{\sum_{x_{i} \in \mathcal{N}\left(x_{o}\right)} S\left(x_{o}, x_{i}\right) L_{x_{i}}}{\sum_{x_{i} \in \mathcal{N}\left(x_{o}\right)} S\left(x_{o}, x_{i}\right)}
$$

where $\mathcal{N}\left(x_{o}\right)$ is the $(2 R+1) \times(2 R+1)$ neighboring patch of $x_{o}$.

Fig. 6 shows an example of the detection result of the Teapot scene. The patch in (a), (b), (c) corresponds to edge, structured texture, and unstructured texture, respectively. The unstructured signal shown in the last column represents the difference between the original and predicted (structured) signals. In the last column, a difference of zero is represented by black, and a difference of 255 is represented by white. For the patches in (a), (b) and (c), the structure information is well predicted, and the unstructured details are not predicted. The unstructured signals show that the structured patches in (a) and (b) can be well distinguished from the unstructured patch in (c). 


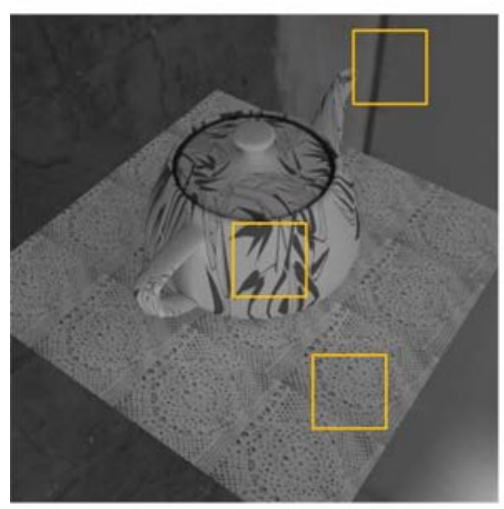

(a)
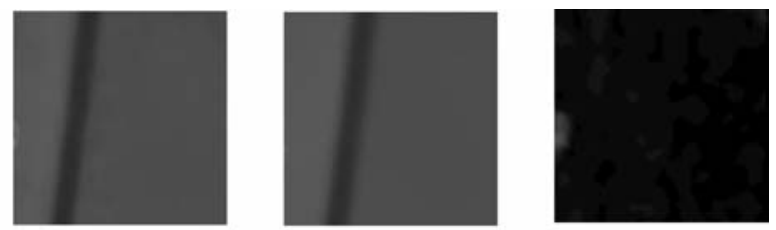

(b)

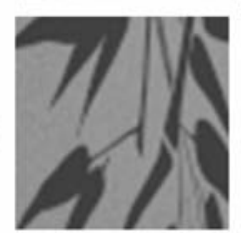

(c)

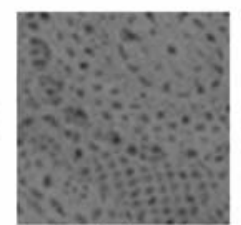

Original

Signal
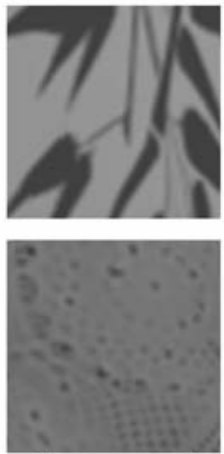

Structured Signal
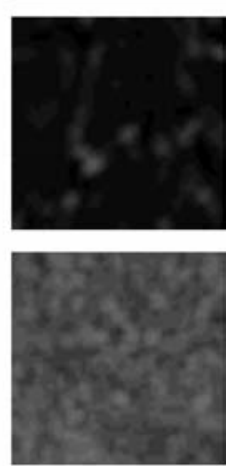

Unstructured

Signal

Figure 6: Detection results of unstructured regions. (a), (b) and (c) correspond to the three regions in the rendered image.

In the proposed method, the computed unstructured signals are used as the entropy masks, which indicate the masking effect of each pixel. Fig. 7 shows the comparison of the masks computed by different visual masking schemes. In the masks in (b) - (d), the structured texture on the poterie is computed with high values, which means the estimated masking effect of the texture is high. The mask shown in (b) is generated by Shannon Entropy. We can see that Shannon Entropy is not able to distinguish regular texture from irregular texture. The mask shown in (c) is generated by the method [14]. In the mask, the pot has higher values than the background. The reason for such kind of result is that the method [14] is based on JPEG compression standard, and it always generates high values for high-frequency regions, including edges, and rapid changing regular/irregular regions. The mask shown in (d) is generated by the method [15]. In (d), the color of the poterie is warmer than that of the background, which means the computed masking effect of the poterie is stronger than the background. The method [15] is based on the contrast sensitivity function and contrast masking, and it yields high masking strength on high-contrast regions, such as edges and high-contrast texture on the poterie. In the mask in (e), the structured texture has low 


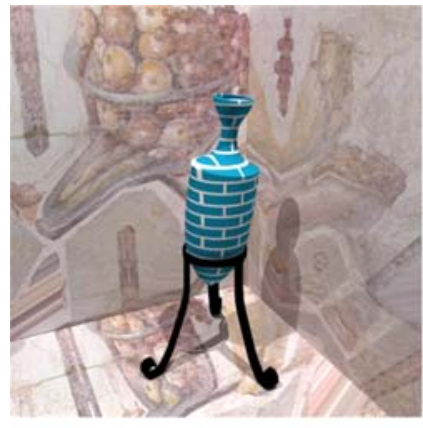

(a)

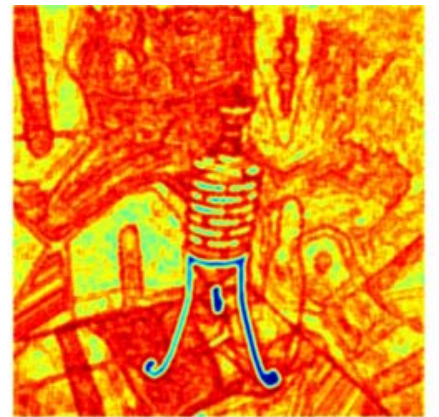

(b)

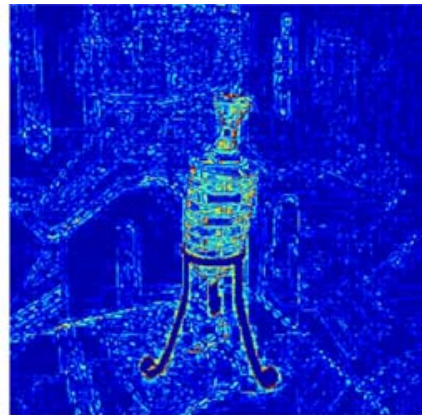

(c)

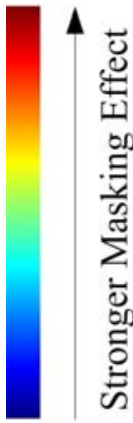

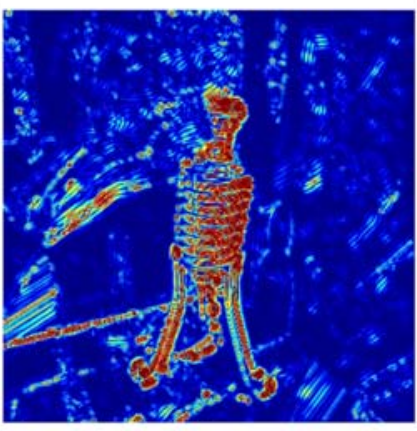

(d)

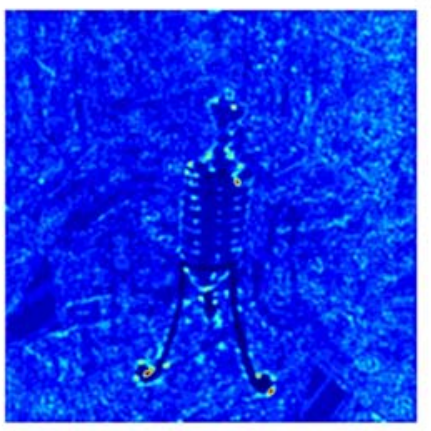

(e)

Figure 7: Comparison of the visual masks computed by different visual masking schemes. (a) is the rendered image. (b), (c), (d), (e) is the visual mask computed by Shannon Entropy, the method [14], the method [15] and the proposed method, respectively. In the visual masks, a warmer color indicates stronger masking effect.

values, while unstructured regions in the background have high values.

To evaluate the performance of the proposed formulation of entropy masking, we inject noise into images according to the computed entropy masks, and compare the distorted images by different visual masking schemes. The noise added into the original image is measured by PSNR, and higher PSNR values indicate that more noise is added into the image. Fig. 8 illustrates the distorted images which contain similar amount of noise (In fact, the amount of noise added in (b)-(d) is slightly less than that in (e)). The Shannon entropy is calculated based on the histogram of the luminance values, and thus Shannon entropy based masking scheme yields high values for regions with luminance change. In the image in (b), visible noise can be detected on regions with illumination variation, such as the orange ball and the white ball. The structured regions (e.g. edges of the balls, numbers on the balls) 


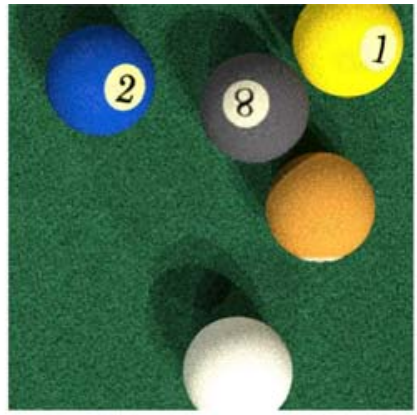

(b) $29.6 \mathrm{~dB}$

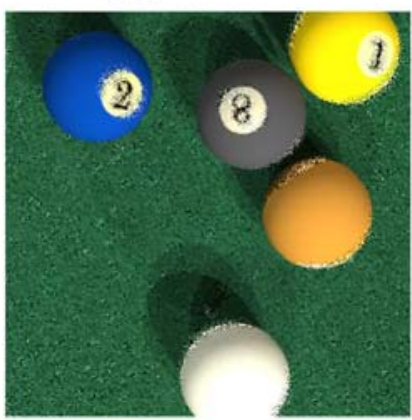

(d) $31.7 \mathrm{~dB}$

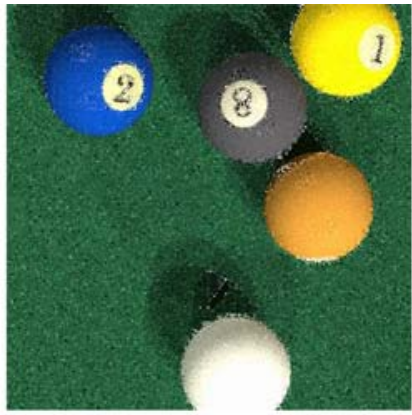

(c) $29.9 \mathrm{~dB}$

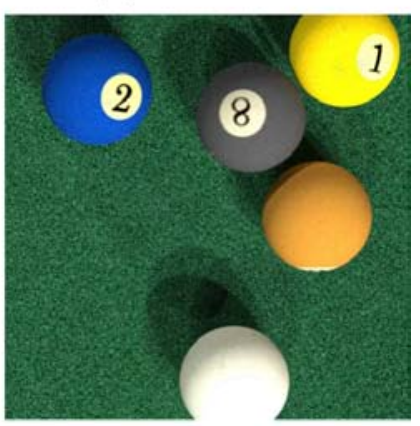

(e) $29.1 \mathrm{~dB}$

Figure 8: Comparison of the distorted images by different visual masking schemes. (a) is the rendered image. (b), (c), (d), (e) is the distorted image by adding noise according to the visual mask computed by Shannon Entropy, the method [14], the method [15] and the proposed method, respectively.

in (c)-(d) contain visible noise since the computed masking effect on these structured regions is strong according to the methods in [14] and [15]. The example shows that noise is less visible by using the visual masks computed by the proposed method.

\subsection{Discussion on parameters}

In the implementation, the neighborhood size $R$ and the local patch size $r$ are two important parameters. The neighborhood size $R$ is selected based on the psychophysical findings that the visual acuity is the highest in foveal field of view, i.e. the small, 1 to 2 degree region at the center of the visual field [25]. In the experiments, the size of the display is $19.1 \mathrm{inch}$, and the display resolution is set to $1440 \times 900$. A recommended ratio of the viewing distance to the image height is in the range of 3 to 6 [26]. We choose a ratio 


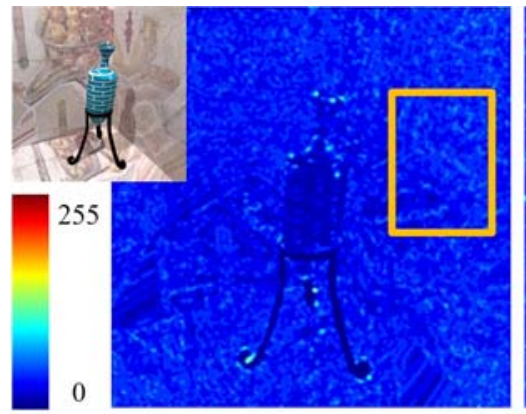

(a) $3 \times 3$

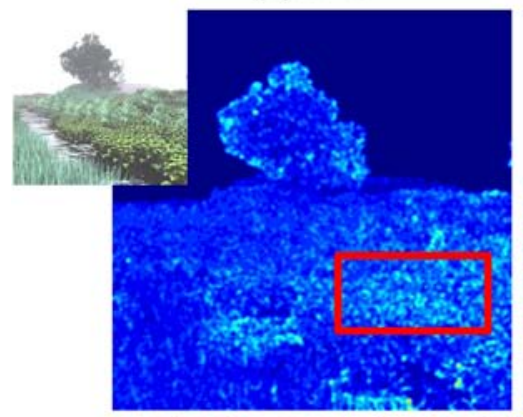

(d) $3 \times 3$

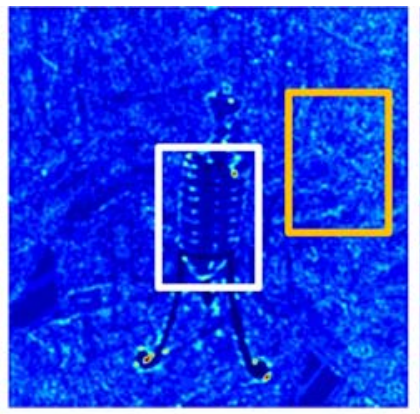

(b) $7 \times 7$

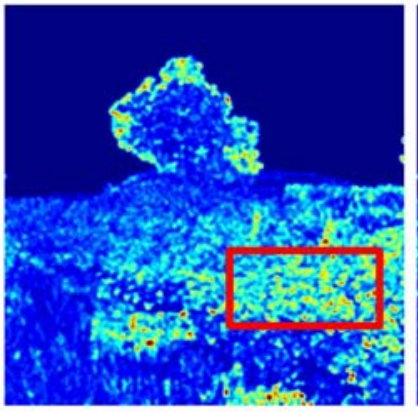

(e) $7 \times 7$

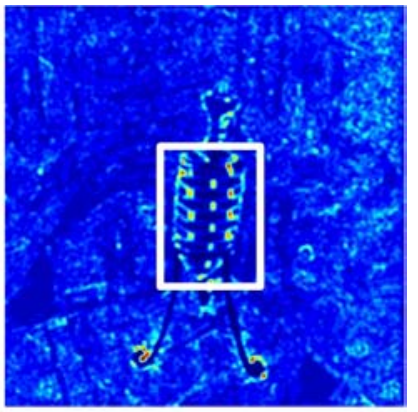

(c) $13 \times 13$

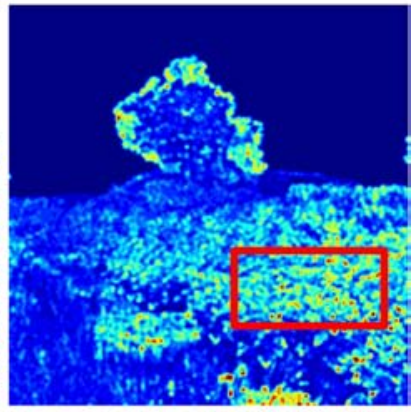

(f) $13 \times 13$

Figure 9: Comparison of different local patch sizes. The top row corresponds to the Poterie scene and the bottom row corresponds to the Plants scene. (a)-(f) are the entropy masks computed by using $3 \times 3,7 \times 7$ and $13 \times 13$ local patches.

of 3 so that the viewers can have a clearer view of the images in the display. The height of each test image is 400 pixels. Based on the parameters, the computed viewing distance is $34 \mathrm{~cm}$, and the pixels per degree (ppd) of visual field is 22 . In the implementation, $R$ is selected to be 11 pixels so that the neighborhood size $(2 R+1) \times(2 R+1)$ corresponds to roughly 1 degree of visual field, where the visual acuity is the highest.

To determine the local patch size $r$, we have conducted experiments by using different patch sizes. If the local patch size $r$ is too large, it would be difficult to find matched patches for fine structure, and pixels in such structured regions will not be well predicted. If $r$ is too small, spatial information around each pixel cannot be captured by the local patches, and unstructured regions cannot be distinguished from structured regions. In the experiment, we have tried $r=1, r=3$ and $r=6$ patch sizes under a neighborhood size $R=11$. Fig. 9 shows the difference maps between an original image and the 
predicted images at different patch sizes. We show the false-colored version of each map for better representation. In the maps, a warmer color indicates a higher difference. It can be observed that with $3 \times 3$ local patches, some unstructured regions are computed with weak masking effect (i.e. in dark blue color), such as the region highlighted with orange color in the Poterie scene and the region highlighted with red color in the Plants scene. With $13 \times 13$ local patches, however, some structured regions, e.g. the structured texture highlighted with white rectangles in the Poterie scene, are given strong masking effect in the masks. In the implementation, we used a local patch size $r=3$.

\subsection{Perceptually based adaptive sampling}

We design a new adaptive sampling scheme based on the proposed entropy masking formulation. The main idea of the perceptual adaptive sampling scheme is to decrease total number of samples without degrading the visual quality of rendered images by distributing fewer samples to unstructured image regions. The proposed adaptive sampling scheme iteratively distributes new samples until the sample budget exhausts. Each iteration is composed of four steps: computing sample values, estimating noise map, computing priority map, and adaptively distributing new samples. In each iteration, a proportion of the sample budget is distributed adaptively according to the priority map, which is a combination of the error map and the entropy mask.

Fig. 10 shows the block diagram of the proposed adaptive sampling scheme. First, a rough image is generated as a quick estimation of the final image. Rough image can be generated by using the GPU rendering hardware [27], and direct illumination [17]. In this work, we generate the rough images by computing the direct illumination using the PBRT rendering engine. We sample the image plane uniformly with a small number of initial samples at every pixel. Then a rough image is rendered by computing the primary rays of the sample rays (i.e. one sample ray experiences at most one bounce from the camera to light sources), as an estimation of the final image.

The global illumination values of the initial sample rays are computed using the path tracing algorithm. Then the per-pixel noise level is computed by the variance:

$$
\varepsilon_{x}=\sqrt{\frac{1}{N_{x}} \sum_{i \in A(x)}\left(s_{i}-\bar{s}_{x}\right)^{2}}
$$

where $\varepsilon_{x}$ is the estimated error of pixel $x$, and $N_{x}$ is the number of samples 


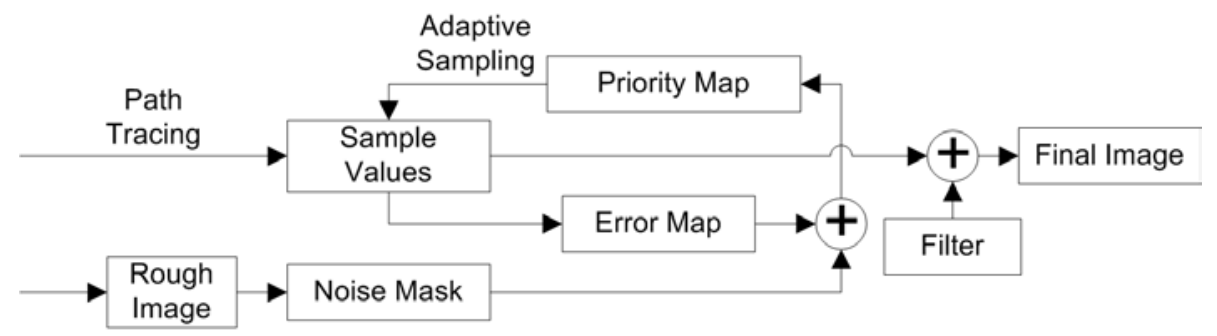

Figure 10: Block diagram of the proposed adaptive sampling scheme in path tracing.

within the region $A(x)$ in the pixel; $s_{i}$ is the value of a sample for this pixel, and $\bar{s}_{x}$ is the average sample value of all the samples for pixel $x$. The estimated noise level of each pixel is stored in a noise map, and higher error means more samples are needed.

To exploit entropy masking in adaptive rendering, a priority map is generated as a combination of the entropy mask and the noise map. Since unstructured regions have stronger masking effect, fewer numbers of samples are needed. The final priority map is calculated as:

$$
O=E \cdot \exp \left(-\frac{M}{2 \gamma^{2}}\right)
$$

where $O$ is the priority map, $E$ is the noise map, and $M$ is the entropy mask; $\gamma$ is a parameter which controls the decay rate of $M$. With a large $\gamma$, the noise map would dominate the sample distribution, and the entropy masking effect would not be considered. With a small $\gamma$, the mask map would play a dominant role, but regions with large rendering noise would not be given sufficient samples and rendering error occurs. The sample distribution maps of the Room scene with different $\gamma$ values are illustrated in Fig. 11. As $\gamma$ decreases from 3 (Fig. 11(b)) to 0.8 (Fig. 11(d)), the influence of the mask map increases, i.e. fewer samples are distributed to unstructured regions. However, when $\gamma$ is too small, all the samples will concentrate on structured regions, as shown in Fig. 11(d). In the experiments, we set $\gamma=1.2$.

The scheme allocates an equal proportion of the total sample budget in each iteration, and the per-pixel sample count is proportional to the pixel's priority value from the previous iteration. After the iterative sampling process, a final image is generated from the sampled values using a filter, which computes each output pixel as a weighted sum of nearby samples. 


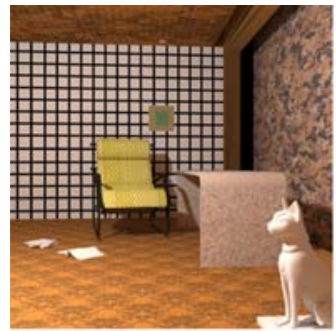

(a)

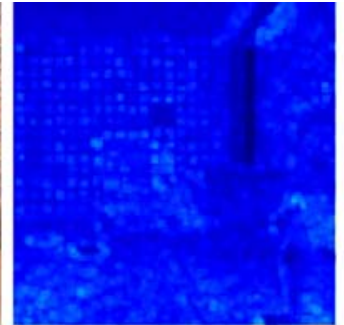

(b)

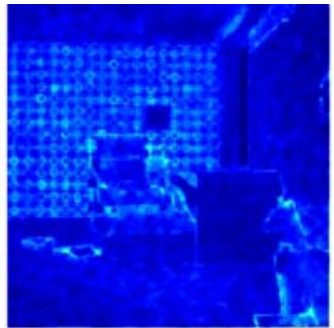

(c)

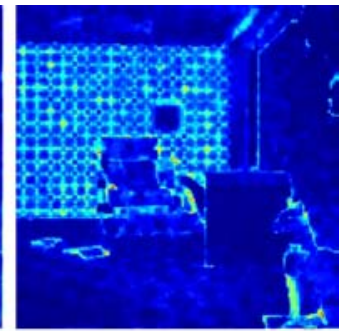

(d)

Figure 11: The effect of different $\gamma$ in sample distribution of the Room scene. (a) is the original image. (b), (c), (d) is sample distribution map at $\gamma=3, \gamma=1.2$ and $\gamma=0.8$, respectively.

Table 1: Cost (in seconds) for the HQ, LQ and TQ images.

\begin{tabular}{l|l|l|l|l|l|l}
\hline & \multicolumn{1}{c|}{ HQ } & \multicolumn{1}{c|}{ LQ } & \multicolumn{4}{c}{ TQ } \\
\hline Scene & $\begin{array}{l}\text { Overall } \\
\text { Time }\end{array}$ & $\begin{array}{l}\text { Overall } \\
\text { Time }\end{array}$ & $\begin{array}{l}\text { Overall } \\
\text { Time }\end{array}$ & $\begin{array}{l}\text { Render } \\
\text { Time }\end{array}$ & $\begin{array}{l}\text { Rough } \\
\text { Image }\end{array}$ & $\begin{array}{l}\text { Mask } \\
\text { Map }\end{array}$ \\
\hline Fairy & 3058.8 & 627.0 & 610.4 & 554.6 & 39 & 16.8 \\
Plants & 4338.3 & 2412.8 & 2355.3 & 2197.4 & 143.8 & 14.1 \\
Poolball & 3150.4 & 1599.1 & 1203.2 & 1186.6 & 3.8 & 12.8 \\
Poterie & 401.2 & 259.6 & 221.7 & 207.1 & 1.3 & 13.3 \\
Room & 1240.7 & 891.3 & 739.9 & 724.5 & 3.5 & 11.9 \\
Sponza & 5497 & 3478.3 & 3334.5 & 3282.0 & 40.1 & 12.4 \\
Teapot & 564.6 & 500.9 & 486.4 & 448.7 & 25.8 & 11.9 \\
Tribal & 518.3 & 366.7 & 319.8 & 280.4 & 27.6 & 11.8 \\
\hline
\end{tabular}

\section{Experimental results}

We compare the proposed method with a recently proposed non-perceptual adaptive sampling method [28]. Three types of images are rendered. Highquality (HQ) images are rendered at high cost by the non-perceptual adaptive sampling method [28]. Test-quality (TQ) images are rendered by the proposed method, but at lower cost. Low-quality (LQ) images are also rendered by the method [28], using the same cost as the TQ images. We cannot make the generation time of TQ and LQ images exactly the same, thus we assign slightly longer time to LQ images, for fair comparison. We record the computational cost (in seconds) of the HQ, LQ and TQ images in Table 1.

We conducted two subjective experiments to demonstrate the advantages of the proposed method. In the first experiment, the participants were shown 
Table 2: Output for a $\chi^{2}$ test

\begin{tabular}{lcc|cc}
\hline & \multicolumn{2}{c}{ HQ and LQ } & \multicolumn{2}{c}{ HQ and TQ } \\
\hline Scene & & & & \\
\hline Fairy & $\chi^{2}=9.000$ & $p=0.003$ & $\chi^{2}=0.000$ & $p=1.000$ \\
Plant & $\chi^{2}=6.250$ & $p=0.012$ & $\chi^{2}=0.250$ & $p=0.617$ \\
Poolball & $\chi^{2}=4.000$ & $p=0.046$ & $\chi^{2}=1.000$ & $p=0.317$ \\
Poterie & $\chi^{2}=9.000$ & $p=0.003$ & $\chi^{2}=0.250$ & $p=0.617$ \\
Room & $\chi^{2}=12.250$ & $p=0.000$ & $\chi^{2}=0.250$ & $p=0.617$ \\
Sponza & $\chi^{2}=12.250$ & $p=0.000$ & $\chi^{2}=0.000$ & $p=1.000$ \\
Teapot & $\chi^{2}=0.250$ & $p=0.617$ & $\chi^{2}=1.000$ & $p=0.317$ \\
Tribal & $\chi^{2}=9.000$ & $p=0.003$ & $\chi^{2}=0.250$ & $p=0.617$ \\
\hline
\end{tabular}

the HQ image together with two test images: one was the HQ image, while the other was the LQ image. In the second experiment, the participants were shown the HQ image together with two test images: the HQ image and the TQ image. In both experiments, the participants were asked to identify the HQ image from the two test images. The purpose of the first experiment is to test whether the participants are able to distinguish the HQ and LQ images, and the purpose of the second experiment is to test whether the participants are able to distinguish the HQ and TQ images.

The proposed adaptive sampling scheme is implemented in the PBRT [1] framework. Eight scenes (Fairy, Plant, Poolball, Poterie, Room, Sponza, Teapot and Tribal) were used in the subjective test. The standard Monte Carlo path tracing algorithm is used for all the test scenes. Test images were rendered with a resolution of $400 \times 400$ pixels, and shown on a 19 -inch LCD monitor with display resolution at $1440 \times 900$ pixels. All the experiments have been performed on a server with Intel X5470 CPU at $3.33 \mathrm{GHz}$ and 34.0GB of RAM.

In each experiment, 16 participants were involved (i.e. in total, 32 participants were involved in these two experiments) and all the 16 participants judge each of the 8 test scenes. Figure 12 shows the experimental results of the first and second sets of viewing tests. If a participant identified the HQ image from these two test images, the result would be marked as "correct"; otherwise, the result would be marked as "incorrect". The percentages of the correct and incorrect results are shown in blue and red bars in Figure 12.

For each pair, a result of $50 \%$ to $50 \%$ selection is the expected result when no noticeable differences exist between the HQ and LQ/TQ images. From 


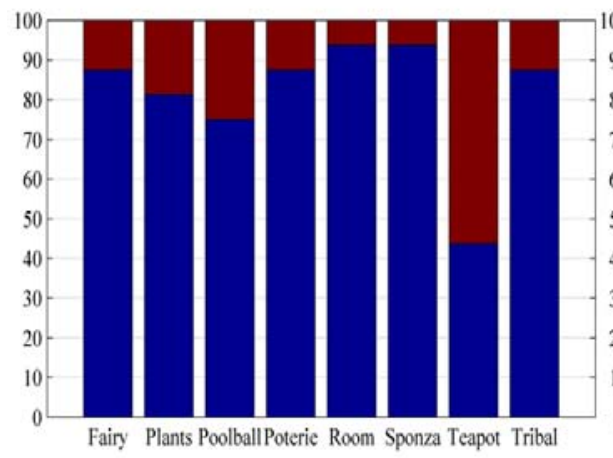

(a)

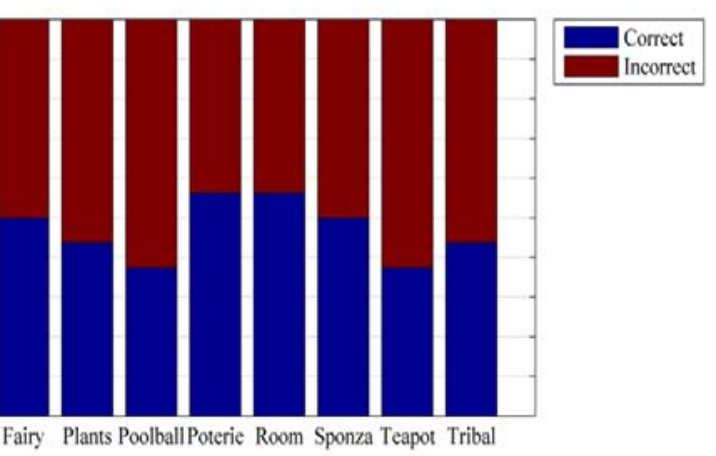

(b)

Figure 12: Test results (a) between the HQ and LQ images, and (b) between the HQ and TQ images.

the results in Figure 12(a), we can see that most participants (81\%) were able to detect the visual difference between the HQ and LQ images. Figure 12(b) shows that about a half of the participants (53\%) were not able to identify the HQ images from the LQ and TQ images. We have further performed statistical analysis on the results of the subjective test. We conducted the $\chi^{2}$ (chi square) test to see whether the number of participants who correctly identified the HQ images occurs by chance (if $p>0.05$ ) or follows a pattern of preference (if $p<0.05$ ).

Table 2 illustrates the results of the $\chi^{2}$ analysis. In this table, $\chi^{2}$ value resembles a normalized sum of squared deviations between the observed and expected frequencies, and $p$ is the probability of getting a number equal or bigger than the observed one. It can be observed that there is a significant difference between the HQ and LQ images by the reference method $(p<$ $0.05)$, except the Teapot scene $(p>0.05)$. It indicates that users have a preference for the HQ images. For the Teapot scene, the user preference from the HQ and LQ images is by chance, since the cost of the LQ image is similar to that of the HQ image. Although fewer number of samples are used in the generation of the TQ image, compared with the HQ image, the overall cost of the TQ image is similar to that of the HQ image. The reason is that the table contains a large amount of unstructured signals while the teapot contains a small amount of unstructured signals, and the proposed method reduces the samples in the table. However, tracing rays hitting on the table costs shorter 
time than tracing rays hitting on the teapot due to the global illumination. Thus, compared with the HQ image, although the sample number decreases in the table, the overall cost does not decrease significantly for the TQ image. As mentioned above, the time assigned to the LQ image is the same as that assigned to the TQ image, and thus is similar to the time assigned to the HQ image, the LQ image is visibly indistinguishable from the HQ image for the scene.

On the other hand, there is no significant difference between the HQ and TQ images from the proposed method $(p>0.05)$. It indicates that the user preference from the HQ and TQ images is by chance. Based on the experimental results in Figure 12 and Table 2, the participants were able to distinguish the HQ and LQ images (except the Teapot scene), but they were not able to distinguish the HQ and TQ images. In other words, the proposed adaptive sampling scheme can yield the same perceived quality as the non-perceptual adaptive sampling scheme [28], with less computational cost.

We also compute the visual difference between the HQ and TQ images using HDR-VDP-2 [29]. HDR-VDP-2 predicts the probability of the differences between two images visible to the human observer. It takes two images as input and generates a map in which each pixel value represents the probability of detecting a pixel difference between these two images by a typical human observer. Note that HDR-VDP-2 does not differentiate unstructured regions from structured ones in the masking computation. However, it can provide useful information on whether or not the visual errors appear on unstructured regions, where the masking effect is strong. For the test scenes, most visual differences (regions with warmer colors in Fig. 13(c) and Fig. 14(c)) reside on regions with unstructured signals. As shown in the results in Fig 12(b), participants in our experiments do not notice the difference for the scenes ( $53 \%$ on average of the participants cannot identify the HQ image from the two bottom images).

We compute the cost reduction by the proposed method and show the results in Table 3. We also decrease the sample number of LQ images, and show the observed threshold generation time (LQ-threshold) when the LQ images are indistinguishable from the HQ images as an additional column in Table 3. The computational reduction ranges from $80 \%$ to $14 \%$ (46\% on average). In general, the computational savings depend on image content, e.g. higher savings can be obtained for a textured scene than a smooth scene. For scenes containing a large amount of unstructured signals, the possible 

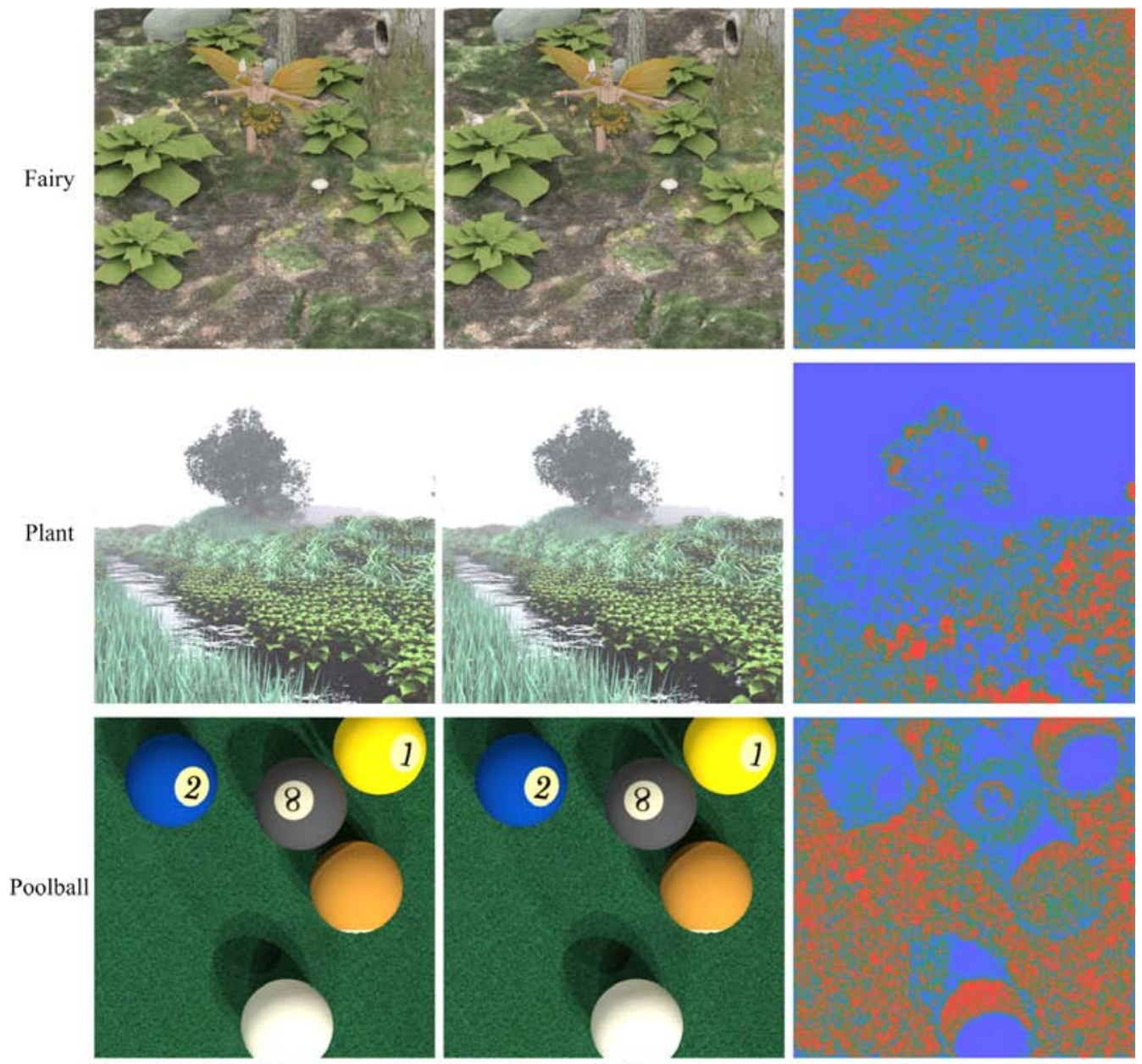

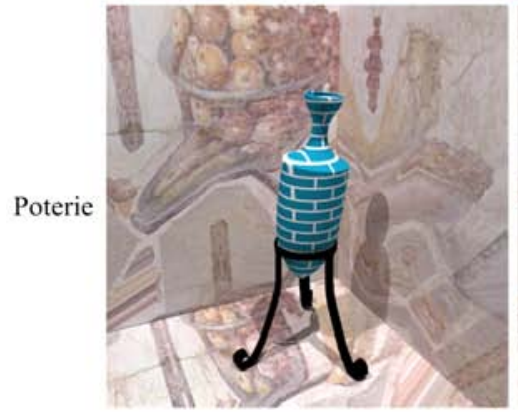

(a)

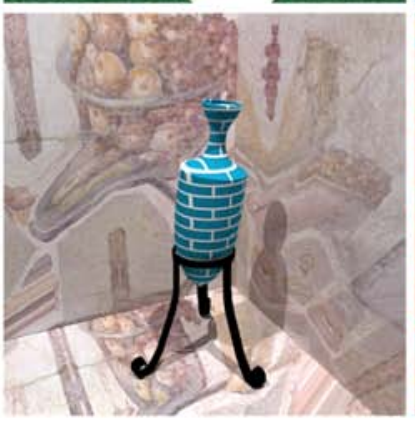

(b)

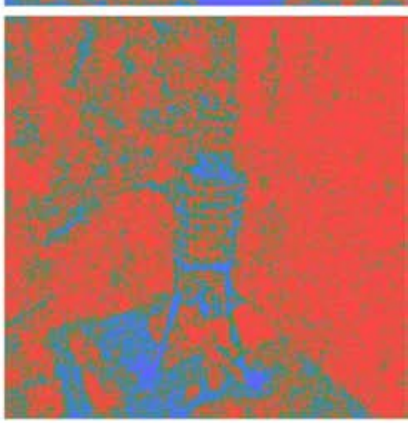

(c)

Figure 13: HDR-VDP-2 visual differences maps (I). (c) illustrates visual difference maps between (a) the HQ images and the (b) TQ images, where warmer color indicates higher possibility that a human observer detects a pixel difference between the HQ and TQ images. 

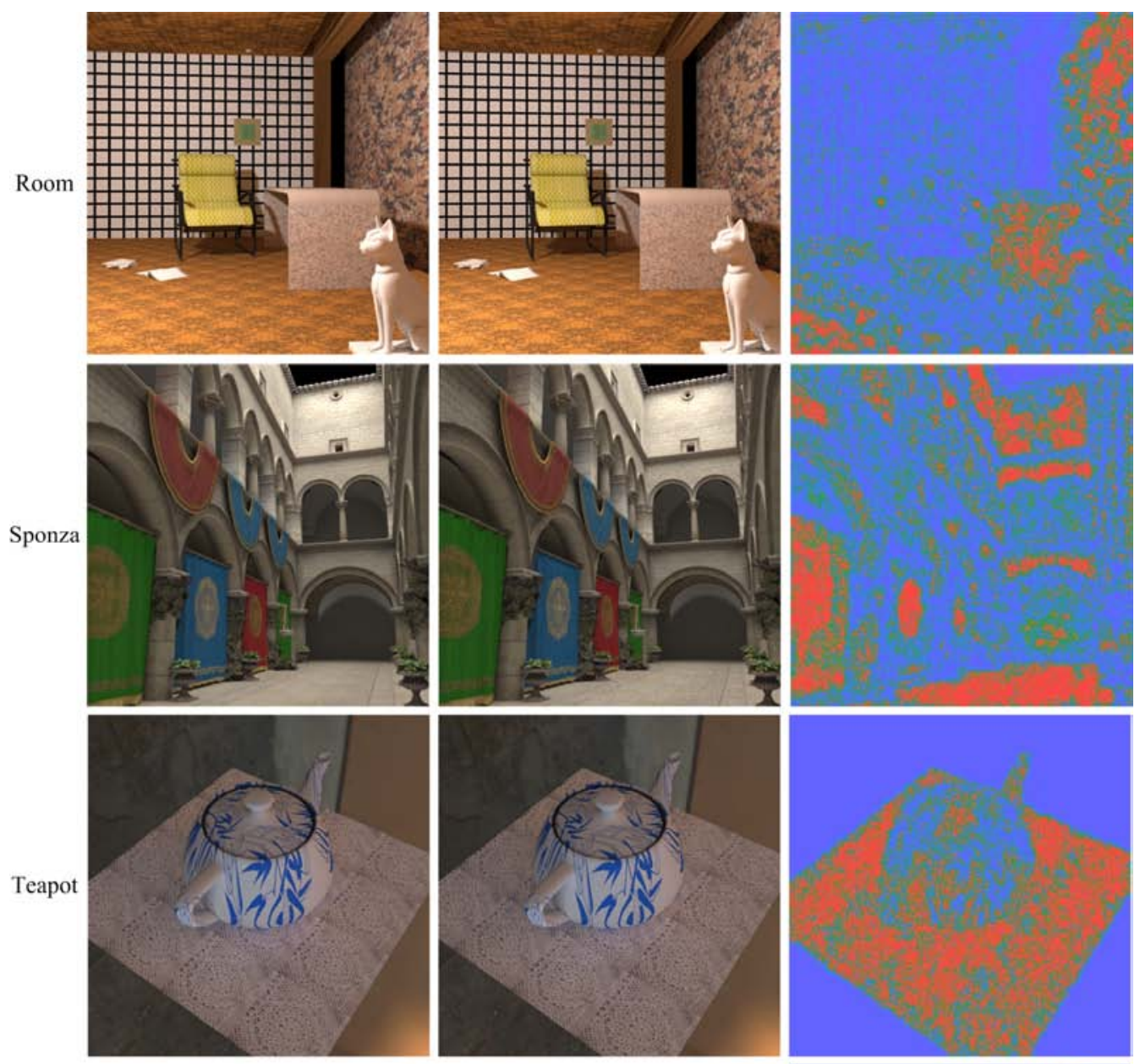

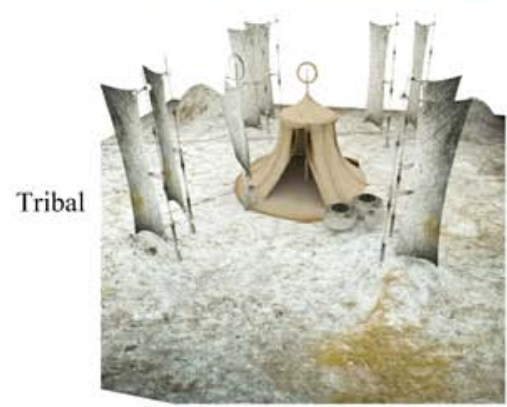

(a)

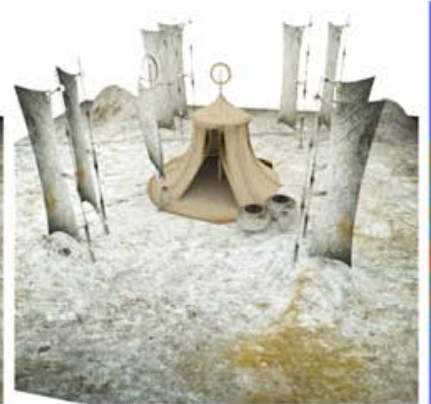

(b)

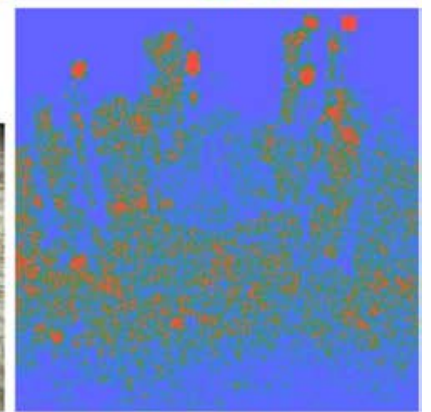

(c)

Figure 14: HDR-VDP-2 visual differences maps (II). (c) illustrates visual difference maps between (a) the HQ images and the (b) TQ images. 
Table 3: Cost (in seconds) reduction by TQ images.

\begin{tabular}{l|l|l|l|l}
\hline & \multicolumn{1}{|c|}{ HQ } & \multicolumn{2}{|c|}{ TQ } & LQ-threshold \\
\hline Scene & $\begin{array}{l}\text { Overall } \\
\text { Time }\end{array}$ & $\begin{array}{l}\text { Overall } \\
\text { Time }\end{array}$ & $\begin{array}{l}\text { Cost } \\
\text { Reduction }\end{array}$ & $\begin{array}{l}\text { Overall } \\
\text { Time }\end{array}$ \\
\hline Fairy & 3058.8 & 610.4 & $80 \%$ & 1785.0 \\
Plant & 4338.3 & 2355.3 & $46 \%$ & 3461.9 \\
Poolball & 3150.4 & 1203.2 & $62 \%$ & 2087.9 \\
Poterie & 401.2 & 221.7 & $45 \%$ & 317.6 \\
Room & 1240.7 & 739.9 & $40 \%$ & 900.8 \\
Sponza & 5497 & 3334.5 & $39 \%$ & 4986.3 \\
Teapot & 564.6 & 486.4 & $14 \%$ & 500.9 \\
Tribal & 518.3 & 319.8 & $38 \%$ & 378.0 \\
\hline
\end{tabular}

decrease of rendering cost is large due to the strong entropy masking effect. For other scenes without unstructured content, the possible decrease of rendering cost is small. We have not categorized the scene content strictly, but in general, we think that the proposed method is more suitable to be used in scenes containing a large amount of unstructured signals. In addition, the time spent on tracing a sample ray varies at different locations on an image, e.g. tracing a ray which hits the light source after a few rebounds takes longer time than tracing a ray that does not intersect an object. The worst case occurs on the Teapot scene, and the costs of the HQ and TQ images are almost the same. The best case occurs on the Fairy scene, with a cost reduction at $80 \%$. The reason is that this scene contains a lot of unstructured regions. As can be seen, exploiting entropy masking in adaptive image rendering leads to computational savings without deteriorating the perceived quality of resultant images.

In this paper, we propose a $2 \mathrm{D}$ based method to estimate the entropy masking effect of rendered images and guide adaptive rendering by exploiting the masking effect. However, there are discussions on whether 2D based computation is sufficient to evaluate 3D scenes [30]. In the future work, we will investigate how to use the 3D attributes, e.g. polygon normal vector and model geometry, to estimate the visual masking effect of rendered images. 


\section{Conclusion}

We have designed a new entropy masking model and implemented a corresponding method to detect unstructured visual signals with strong entropy masking effect. In existing entropy masking schemes, the spatial correlation of neighboring pixels is not considered, and structured regions cannot be well distinguished from unstructured regions. In structured regions, pixels are highly correlated with their neighboring pixels, and neighboring pixels with similar local patterns to the center pixel can be detected. In the proposed scheme, each pixel is predicted from neighboring pixels with similar local structure, and the difference between the original pixel and the predicted pixel is considered as the unstructured visual signal. We have incorporated the entropy masking scheme into an iterative adaptive sampling algorithm: the allocation of samples is guided by both the estimated noise level and the estimated level of masking of image content. Experimental results have demonstrated that the proposed perceptual image rendering method can obtain much time-saving without reducing perceived quality compared with the existing adaptive sampling methods.

\section{Acknowledgement}

We are grateful for the editor and two anonymous reviewers' insightful comments that have prompted us for many rounds of re-thinking, re-work and re-positioning of our research. We would also like to thank the creators of the models used in this paper: Fairy forest scene (Ingo Wald) via the Utah 3D Animation Repository; Crytek Sponza (Marko Dabrovic and Frank Meinl) via the AIM@SHAPE repository; tribal site (Viviano), downloaded from artist-3d.com; Poolball (Toshiya Hachisuka); Plant (Deussen et al.) and Teapot from the PBRT2 book; and Poterie (user 3dsud of www.exchange3d.com). This work was supported in part by the Ministry of Education (MoE) AcRF Tire 1 Funding, Singapore, under Grant 2014-T1002-068. The project is also sponsored by the scientific research foundation for the returned overseas Chinese scholars, state education ministry.

[1] M. Pharr, G. Humphreys, Physically Based Rendering: From Theory to Implementation, Morgan Kaufmann, 1 and 762, 2010.

[2] D. Bartz, D. Cunningham, J. Fischer, C. Wallraven, State-of-the-Art of the Role of Perception for Computer Graphics, in: Proceedings of the 29th Annual Conference Eurographics EG 2008, vol. 4, 65-86, 2008. 
[3] S. Daly, Digital images and human vision, chap. The visible differences predictor: an algorithm for the assessment of image fidelity, MIT Press, Cambridge, MA, USA, 192, 1993.

[4] P. L. C. A. Ninassi, O. Le Meur, D. Barba, On the performance of human visual system based image quality assessment metric using wavelet domain, SPIE proceedings Human Vision and Electronic Imaging XIII 6806.

[5] G. E. Legge, J. M. Foley, Contrast masking in human vision, Journal of the Optical Society of America 70 (12) (1980) 1458-1471.

[6] A. B. Watson, R. Borthwick, M. Taylor, Image Quality and Entropy Masking, Human Vision, Visual Processing, and Digital Display VIII 3016 .

[7] M. P. Eckert, A. P. Bradley, Perceptual quality metrics applied to still image compression, Signal Processing 70 (3) (1998) 177 - 200.

[8] A. H. Sadr, S. Ghaemmaghami, Robustness enhancement of contentbased watermarks using entropy masking effect, in: Proceedings of the 4th international conference on Digital Watermarking, IWDW'05, 444458, 2005.

[9] S. P. Maity, M. K. Kundu, DHT domain digital watermarking with low loss in image informations, AEU - International Journal of Electronics and Communications 64 (3) (2010) $243-257$.

[10] C. E. Shannon, W. Weaver, A mathematical theory of communication, 1948.

[11] I. Cheng, P. Boulanger, A Visual Quality Prediction Model for 3D Texture, in: EUROGRAPHICS, 101-104, 2005.

[12] M. R. Bolin, G. W. Meyer, A perceptually based adaptive sampling algorithm, in: Proceedings of the 25th annual conference on Computer graphics and interactive techniques, SIGGRAPH '98, 299-309, 1998.

[13] H. Yee, S. Pattanaik, D. P. Greenberg, Spatiotemporal sensitivity and visual attention for efficient rendering of dynamic environments, ACM Transactions on Graphics (TOG) 20 (1) (2001) 39-65. 
[14] B. Walter, S. N. Pattanaik, D. P. Greenberg, Using perceptual texture masking for efficient image synthesis, Computer Graphics Forum 21 (3) (2002) 393-399.

[15] L. Qu, G. W. Meyer, Perceptually guided polygon reduction, Visualization and Computer Graphics, IEEE Transactions on 14 (5) (2008) $1015-1029$.

[16] J. A. Ferwerda, P. Shirley, S. N. Pattanaik, D. P. Greenberg, A model of visual masking for computer graphics, in: Proceedings of the 24th annual conference on Computer graphics and interactive techniques, 143-152, 1997.

[17] M. Ramasubramanian, S. N. Pattanaik, D. P. Greenberg, A perceptually based physical error metric for realistic image synthesis, in: Proceedings of the 26th annual conference on Computer graphics and interactive techniques, SIGGRAPH '99, 73-82, 1999.

[18] K. Myszkowski, P. Rokita, T. Tawara, Perceptually-informed accelerated rendering of high quality walkthrough sequences, in: Proceedings of the Tenth Eurographics Workshop on Rendering, 5-18, 1999.

[19] K. Myszkowski, P. Rokita, T. Tawara, Perception-Based Fast Rendering and Antialiasing of Walkthrough Sequences, IEEE Transactions on Visualization and Computer Graphics 6 (2000) 360-379.

[20] K. Myszkowski, T. Tawara, H. Akamine, H.-P. Seidel, Perception-guided global illumination solution for animation rendering, in: Proceedings of the 28th annual conference on Computer graphics and interactive techniques, SIGGRAPH '01, 221-230, 2001.

[21] W. B. Thompson, R. W. Fleming, S. H. Creem-Regehr, J. K. Stefanucci, Visual perception from a computer graphics perspective, chap. II Building Blocks, 2.1 Visual Sensitivity and III Surfaces And Movement, 3.3 Illumination, Shading, and Shadows, CRC Press, 2011.

[22] G. Lavoué, A Local Roughness Measure for 3D Meshes and Its Application to Visual Masking, ACM Transactions on Applied Perception 5 (4) (2009) 21:1-21:23. 
[23] K. Wang, F. Torkhani, A. Montanvert, A fast roughness-based approach to the assessment of 3D mesh visual quality, Computers and Graphics 36 (7) (2012) 808-818.

[24] L. Váša, J. Rus, Dihedral Angle Mesh Error: a fast perception correlated distortion measure for fixed connectivity triangle meshes, in: Computer Graphics Forum, vol. 31, Wiley Online Library, 1715-1724, 2012.

[25] V. Bruce, P. R. Green, M. A. Georgeson, Visual perception: Physiology, psychology, \& ecology, Psychology Press, 2003.

[26] I. Recommendation, 500-11, Methodology for the subjective assessment of the quality of television pictures, International Telecommunication Union, Geneva, Switzerland 4 (2002) 2.

[27] P. Longhurst, K. Debattista, A. Chalmers, A GPU based saliency map for high-fidelity selective rendering, in: Proceedings of the 4th international conference on Computer graphics, virtual reality, visualisation and interaction in Africa, 21-29, 2006.

[28] N. K. Kalantari, P. Sen, Removing the Noise in Monte Carlo Rendering with General Image Denoising Algorithms, Computer Graphics Forum (Proceedings of Eurographics 2013) 32 (2).

[29] R. Mantiuk, K. J. Kim, A. G. Rempel, W. Heidrich, HDR-VDP-2: a calibrated visual metric for visibility and quality predictions in all luminance conditions, ACM Trans. Graph. 30 (4) (2011) 40:1-40:14.

[30] B. E. Rogowitz, H. E. Rushmeier, Are image quality metrics adequate to evaluate the quality of geometric objects?, in: Photonics West 2001Electronic Imaging, International Society for Optics and Photonics, 340348, 2001. 